\title{
Improved stochastic linearization method using mixed distributions
}

\author{
Jorge E. Hurtado a, Alex H. Barbat ${ }^{\mathrm{b}, *}$

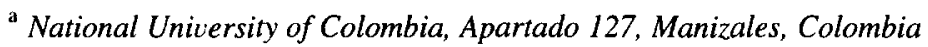 \\ ${ }^{\mathrm{b}}$ Technical University of Catalonia, c/ Gran Capitán, s/n, Barcelona 08034, Spain
}

\begin{abstract}
A new procedure for the random vibration analysis of hysteretic structures using stochastic equivalent linearization is reported. Its aim is to improve the prediction of the response obtained by conventional Gaussian linearization technique. To this purpose, mixed discrete-continuous Gaussian distributions are used taking into account the bounded nature of the non-linear restoring force. The simple but important property of the mixed distribution is its linearity, which allows the use of the previous results obtained by the Gaussian hypothesis, avoiding the need of employing non-Gaussian continuous distributions or other time-consuming techniques such as local Monte Carlo simulations. Closed-form expressions of the new linearization coefficients for the Bouc-Wen-Baber model are then provided. The relative weights of the discrete and Gaussian distributions are calculated in dependence of the degree of non-linearity in each time step. The comparison of the results with previously published ones obtained by simulation shows a good agreement, providing a substantial improvement of the method with respect to the conventional Gaussian technique with the same calculation effort. Copyright (C) 1996 Elsevier Science Ltd.
\end{abstract}

Keywords: Stochastic equivalent linearization; Random vibrations; Non-Gaussian distributions; Hysteretic systems; Bouc-Wen-Baber hysteretic model

\section{Introduction}

The method of stochastic equivalent linearization has received increasing attention during the last two decades by scientists and engineers dealing with the analysis of random vibration of mechanical and structural systems. Since its initial developments by Caughey [1], Iwan [2] and others, the method received its major impulse for practical applications when Atalik and Utku [3] showed that for Gaussian distributions the calculation of the linearization matrices can be done in a much simpler way than for other distributions. Since then the method has been extensively applied in structural engineering for the prediction of response statistics and it is actually considered as the most versatile for the random vibration analysis of non-linear structures [4]. For a detailed description of the method see [5].

\footnotetext{
" Corresponding author.
} 
In the field of earthquake engineering the hysteretic model presented by Bouc [6] and developed by Wen [7] and Baber and Wen [8] has been used in random vibration analysis of reinforced concrete and steel structures [9], soil profiles [10], base isolated buildings [11] and hybrid control [12]. It has been demonstrated that the model does not completely agree with plasticity theory and errors in the prediction of the deterministic response occur especially when the restoring force does not change sign in a cycle - a case in which the model produces negative energy dissipation [13]. Nevertheless, for random vibration purposes the model has been widely accepted due to its versatility and the possibility of expressing the linearization coefficients in a closed form. Moreover, the local violations of the plasticity principles are of no importance in random vibration analyses if the expected value of the restoring force is zero, as is the case normally assumed in practical applications, and in this case the dissipated energy will always be positive. Although, some modifications have been introduced in the model to correct this anomalous behavior and linearization coefficients have been calculated accordingly [14].

However, an important deficiency has been found in the method of equivalent linearization when using this and other hysteretic models under the hypothesis of Gaussian response; namely, that it produces an underestimation of the standard deviation of the displacement response that can reach values of the order of 30 or $40 \%$, with the consequence that the prediction of failure probabilities deviate greatly from the simulation results, specially at high levels of excitation. This is due to the fact that a Gaussian behavior is assumed for all variables, while physically the restoring force is constrained to lie in a finite region.

As a consequence, several attempts have been made in the last years to overcome this difficulty. Important efforts in this direction have been exerted by H.J. Pradlwarter, G.I. Schue̊ller and coinyestigators $[15,16]$ who have proposed some numerical techniques to obtain better response statistics in the general case order to obtain the desired Gaussian variables, Monte case of non-stationary respo
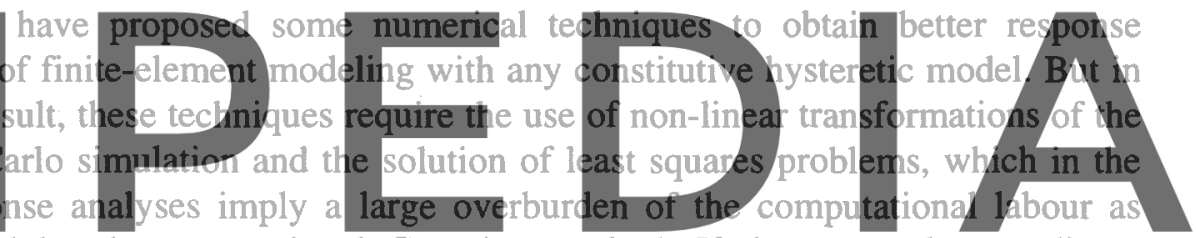

compared with that required by the conventional Gaussian method. If, however, the non-linear

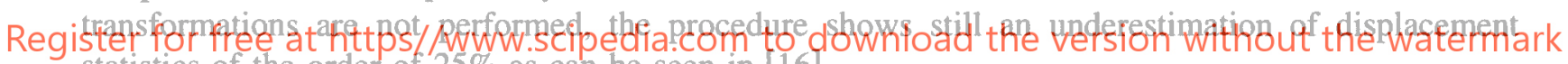
statistics of the order of $25 \%$ as can be seen in [16].

On the other hand, the use of truncated Gaussian plus Dirac pulses proposed recently by Kimura et

al. [17] for an elasto-plastic oscillator is quite accurate, but it requires the calculation of several double integrals for each value of time, which increase the amount of calculations. Finally, an empirical equation proposed by Wen and Yeh [18] has been derived for the special case of biaxial vibration of shear buildings. Since it requires some knowledge about the degree of non-linearity reached by the oscillator in order to calculate a coefficient entering into it, and this coefficient depends also on the location of the story, the correction must be applied a posteriori and its use is, in general, limited to that type of structure. On the contrary, in the method presented in this paper the improved statistics are obtained by a straightforward, general procedure.

The proposed algorithm is a modification of the classical equivalent linearization method. Its purpose is to improve the calculation of the response statistics of non-linear oscillators for which the restoring force is bounded by constant or time-dependent limits (non-degrading and degrading cases, respectively) as predicted by the Gaussian assumption of the response. The method makes use of the mathematical advantages of both the Gaussian and Dirac delta functions. This allows the calculation 
of closed-form expressions for the linearization coefficients in the case of the Bouc-Wen-Baber model, thus preserving the computational simplicity of the conventional Gaussian method. The comparison with simulation results shows a very good improvement in the estimation of statistics of displacement responses.

\section{Proposed method}

\subsection{Equivalent linearization}

The equation of motion of a single-degree-of-freedom system with a mass $m$ and a viscous damping $c$ is given by

$$
m \ddot{u}+c \dot{u}+g(u, \dot{u}, z)=f(t)
$$

where $g(\cdot)$ is the restoring force, $f(\cdot)$ the external excitation, $u$ the displacement of the system. Let the restoring force be expressed as

$$
g(u, \dot{u}, z)=\alpha k u+(1-\alpha) k z
$$

where $k$ is a constant of proportionality having units of stiffness and $z$ a non-linear function with displacement units. In the Bouc-Wen-Baber model $z$ is governed by the following differential equation [8]:

$$
\dot{z}=A \dot{u}-\beta|\dot{u} \| z|^{n-1} z-\gamma \dot{u}|z|^{n}
$$

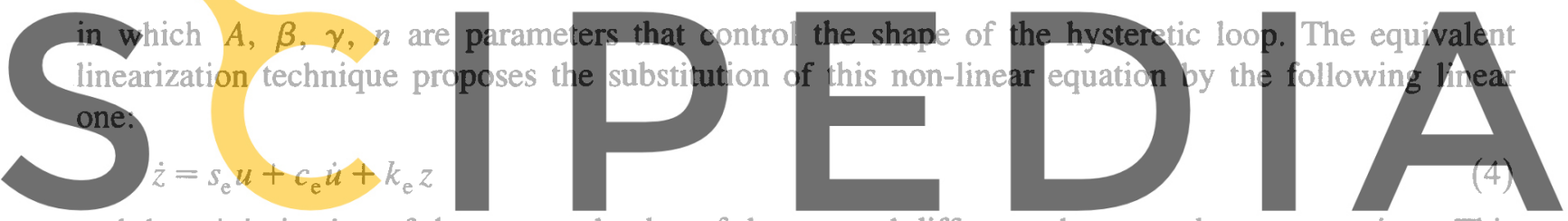

and the minimization of the expected value of the squared difference between the two equations. This

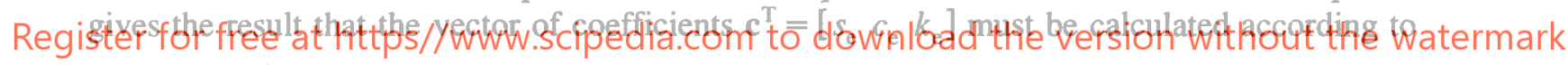

$$
c^{T}=E\left\{\dot{z} \mathbf{y}^{T}\right\} S^{-1}
$$

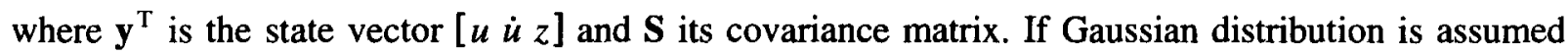
for all the state variables and for their joint behavior, the first term of the left-hand side of the above equation becomes [5]

$$
\mathrm{E}\left\{\dot{z} \mathbf{y}^{\mathrm{T}}\right\}=\mathrm{E}\{\nabla \dot{z}\} \mathbf{S}
$$

so that the vector of coefficients can be calculated as

$$
\mathbf{c}^{\mathrm{T}}=\mathrm{E}\{\nabla \dot{z}\} \text {. }
$$

For the case of the hysteretic model under consideration, the coefficients are expressed as [14]

$$
\begin{aligned}
& s_{\mathrm{g}}=0 \\
& c_{\mathrm{g}}=A-\beta F_{1}-\gamma F_{2} \\
& k_{\mathrm{g}}=-\beta F_{3}-\gamma F_{4}
\end{aligned}
$$


where the subscript e has been changed to $\mathrm{g}$ to denote the Gaussian assumption. In these equations,

$$
\begin{aligned}
& F_{1}=\frac{\sigma_{z}^{n}}{\pi} \Gamma\left(\frac{n+2}{2}\right) 2^{n / 2} I_{\mathrm{s}} \\
& F_{2}=\frac{\sigma_{z}^{n}}{\sqrt{\pi}} \Gamma\left(\frac{n+1}{2}\right) 2^{n / 2} \\
& F_{3}=\frac{n \sigma_{\dot{u}} \sigma_{z}^{n-1}}{\pi} \Gamma\left(\frac{n+2}{2}\right) 2^{n / 2}\left(2\left(1-\rho_{\dot{u} z}^{2}\right)^{(n+1) / 2}+\rho_{\dot{u} z} I_{\mathrm{s}}\right) \\
& F_{4}=\frac{n \rho_{\dot{u} z} \sigma_{\dot{u}} \sigma_{z}^{n-1}}{\pi} \Gamma\left(\frac{n+1}{2}\right) 2^{n / 2}
\end{aligned}
$$

with

$$
\begin{aligned}
& I_{\mathrm{s}}=2 \int^{\pi / 2} \sin ^{n} \theta \mathrm{d} \theta \\
& \ell=\tan ^{-1}\left(\frac{\sqrt{1-\rho_{u z}^{2}}}{\rho_{\dot{u} z}}\right) .
\end{aligned}
$$

The fact mentioned above that the restoring force is bounded suggests the use of the following alternative density function for the variable $z$ :

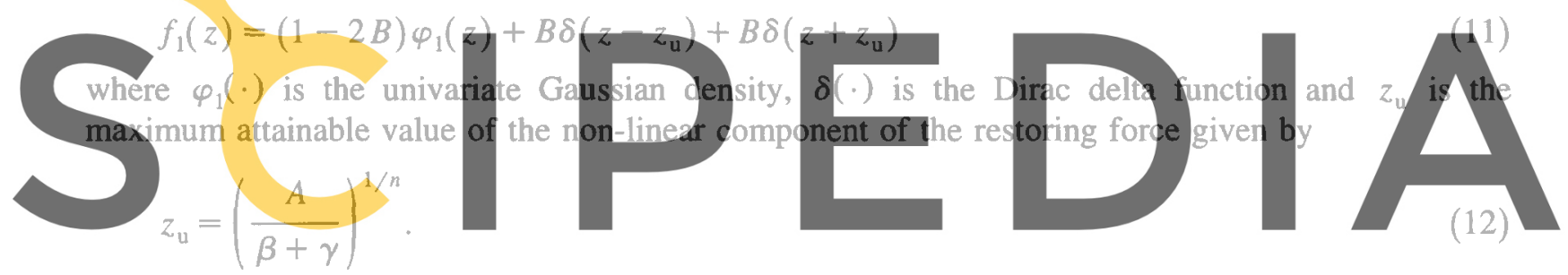

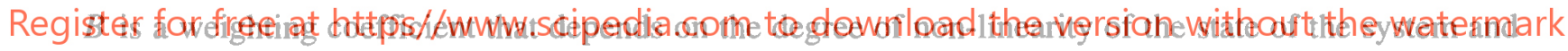
that can be estimated (as will be described later); it becomes a function of time in the non-stationary case. The corresponding joint distribution relating $z$ and the velocity $u$ can be expressed as

$$
f_{2}(u, z)=(1-2 B) \varphi_{2}(\dot{u}, z)+B \delta\left(z-z_{\mathrm{u}}\right) \varphi_{1}(\dot{u})+B \delta\left(z+z_{\mathrm{u}}\right) \varphi_{1}(\dot{u})
$$

where $\varphi_{2}(\cdot, \cdot)$ is the bivariate normal density. A similar equation describes the joint behavior of $z$ and the displacement $u$.

It is evident that this formulation makes use of the mathematical advantages of the Gaussian and Dirac functions, and not only of the latter as is seen in the approach of Kimura et al. [17] who use the two Dirac delta pulses combined with a truncated Gaussian density. Also, the linearity of the proposed mixed density allows the decomposition of the calculation of the coefficients between a purely Gaussian part and a mixed discrete Gaussian one, so that their values in Eq. (8) can be used with the corresponding weights. Thus, the application of the proposed mixed density to the Bouc-Wen-Baber model gives the new values

$$
\mathbf{c}^{\mathrm{T}}=\left[s_{\mathrm{e}} c_{\mathrm{e}} k_{\mathrm{e}}\right]=(1-2 B)\left[s_{\mathrm{g}} c_{\mathrm{g}} k_{\mathrm{g}}\right]+2 B\left[s_{\mathrm{d}} c_{\mathrm{d}} k_{\mathrm{d}}\right] \mathbf{V}^{-1}
$$


where $\mathbf{V}$ is the covariance matrix

$$
\mathbf{V}=\mathrm{E}\left\{\left(\begin{array}{ccc}
u^{2} & u \dot{u} & u z \\
\dot{u} u & \dot{u}^{2} & \dot{u} z \\
z u & z \dot{u} & z^{2}
\end{array}\right)\right\}
$$

and

$$
\begin{aligned}
& s_{\mathrm{d}}=\sigma_{u \dot{u}}\left(A-\gamma z_{\mathrm{u}}^{n}\right) \\
& c_{\mathrm{d}}=\sigma_{\dot{u}}^{2}\left(A-\gamma z_{\mathrm{u}}^{n}\right) \\
& k_{\mathrm{d}}=-\sigma_{\dot{u}} \beta z_{\mathrm{u}}^{n+1} \sqrt{\frac{2}{\pi}} .
\end{aligned}
$$

The hysteretic model under study has been extended to the case of degrading systems in [8]. The above equations also apply to this case with the transformations

$$
\begin{aligned}
A \rightarrow \mu_{A}(t) & =\frac{A}{\mu_{\eta}(t)} \\
\beta \rightarrow \mu_{\beta}(t) & =\frac{\mu_{\nu}(t) \beta}{\mu_{\eta}(t)}
\end{aligned}
$$

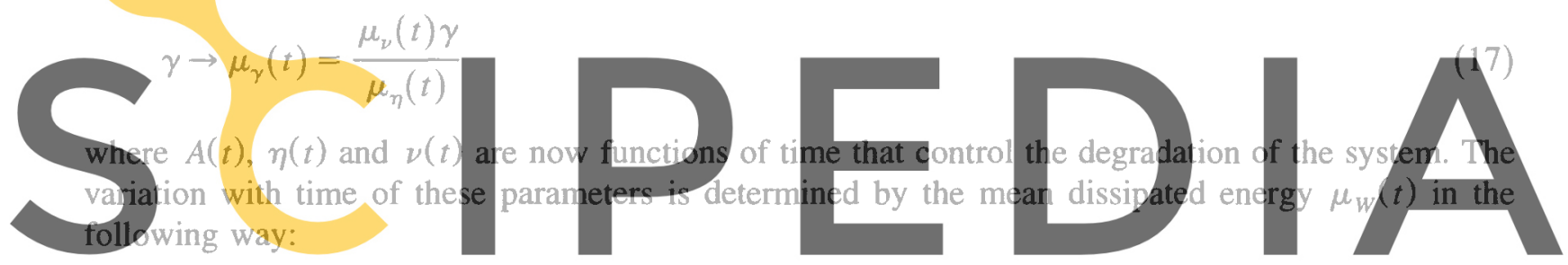

$\begin{aligned} & \mu_{A}(t)=A_{0}-\delta_{A} \mu_{W} \\ & \text { Register for free at https//Www.scipedia.com to download the version without the watermark } \\ & \mu_{\eta}(t)=\eta_{0}+\delta_{\eta} \mu_{W}\end{aligned}$

$$
\mu_{\nu}(t)=\nu_{0}+\delta_{\nu} \mu_{W}
$$

in which the subscript 0 indicates the initial values and the $\delta \mathrm{s}$ are the coefficients of degradation of the respective parameters. It is important to observe that the increase of $\eta$ and $\nu$ causes stiffness or strength degradation, respectively, and that the decrease of $A$ implies both kinds of deterioration. Notice that now the maximum value of the non-linear component that must be used in Eq. (16) becomes also a function of time $z_{\mathrm{u}}(t)$.

\subsection{Weighting function}

The last point which has to be discussed for the implementation of the present method is the appropriate value of the weighting function that must be used in order to improve the estimated statistics of the response. The weight must be such that its value is low for linear behavior and high for a strongly non-linear one. Since the response of the non-linear system up to the yielding value is 
Gaussian under a Gaussian input, it seems logical to take as the weight the probability mass in excess of the maximum value of the non-linear component

$$
B_{0}=\Phi\left(z_{\mathrm{u}}\right)=\int_{-\infty}^{z_{\mathrm{u}}} \varphi_{\mathrm{l}}(z) \mathrm{d} z
$$

which is also the intensity of Dirac functions used in [17] in combination with truncated Gaussian distribution. Since in the present case the Gaussian function is not truncated, there still remains a probability mass above the critical level, so that the improvement obtained by applying this correcting term can be expected to be low. Hence a stronger criterion must be invoked. After testing several alternatives, a weighting function defined as the contribution to the total variance of the area above the critical level $z_{\mathrm{u}}$ was found satisfactory and simple to evaluate. This is given by

$$
B_{2}=\frac{v_{z}^{2}}{\sigma_{z}^{2}}
$$

where

$$
v_{z}^{2}=\int_{z_{u}}^{\infty} z^{2} \varphi_{1}(z) d z
$$

After replacing the Gaussian density function in the above equation, the following expression is obtained:

$$
B_{2}=\frac{1}{\sqrt{\pi}} \Gamma^{c}\left(1.5, \frac{z_{\mathrm{u}}^{2}}{2 \sigma_{z}^{2}}\right)
$$

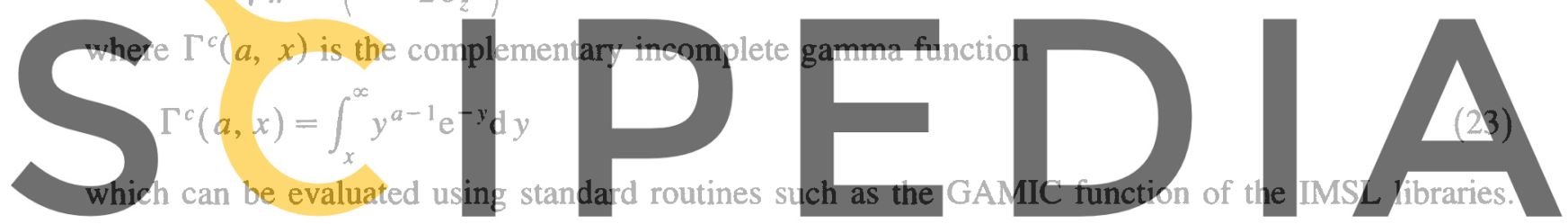

Register for free at https//Wwwy.scipedia.com to download the version without the watermark

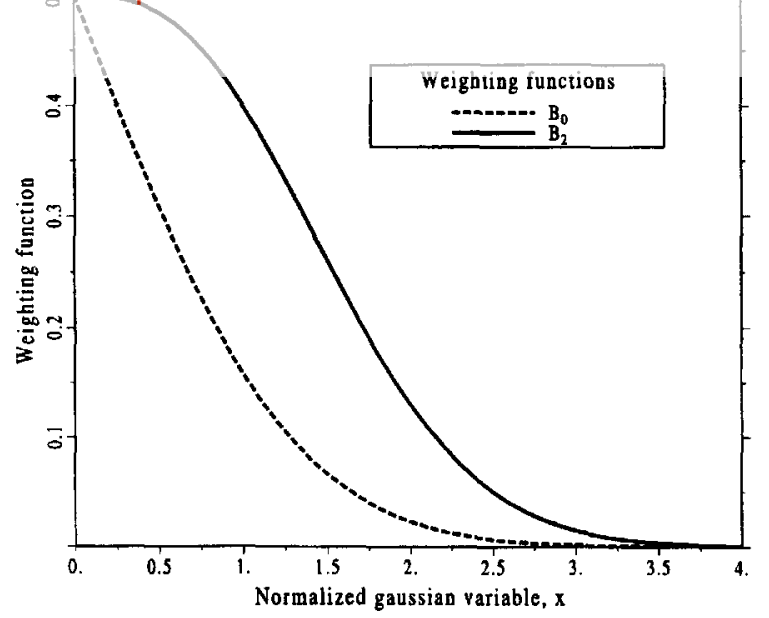

Fig. 1. Values of the weighting function corresponding to probability $\left(B_{0}\right)$ and variance $\left(B_{2}\right)$ criteria. 
Fig. 1 shows the values of the two expressions of the weighting function for a normalized Gaussian variable $x$. It is seen that the two are almost equal when $x \rightarrow 0$ or $x \rightarrow \infty$, corresponding respectively to high and low standard deviation, but they are rather different in the intermediate range. As it will be seen in the following examples, the use of $B_{2}$ gives excellent results for both non-degrading and degrading cases, except in one example where a large strength degradation (governed by a high value of $\delta_{\nu}$ ) takes place. In cases like this it is advisable to use a stronger correction, as it will be shown in the next section.

\section{Numerical study}

Some simple numerical analyses were performed in order to test the goodness of the present method with respect to the conventional technique. The data of the selected examples as well as the simulation results were taken from [8], where the classical Gaussian approach was followed. In all cases the response statistics are obtained by solving the Lyapunov differential equation [5]

$$
\dot{\mathbf{S}}=\mathbf{A} \mathbf{S}+\mathbf{S A}^{\mathrm{T}}+\mathbf{W}
$$

where $\mathbf{S}$ is the covariance matrix of the state vector $\mathbf{y}$ expanded to include the required filter parameters; $\mathbf{A}$ is the state matrix which includes the linearization matrices and $\mathbf{W}$ is a matrix whose only non-zero element is equal to the intensity of the modulated input noise at each time step. All variables collected in the state vector are assumed to have a zero mean, so that their standard deviations will also be designated as root mean square (r.m.s.) responses in what follows.

3.1. Non-degrading simple

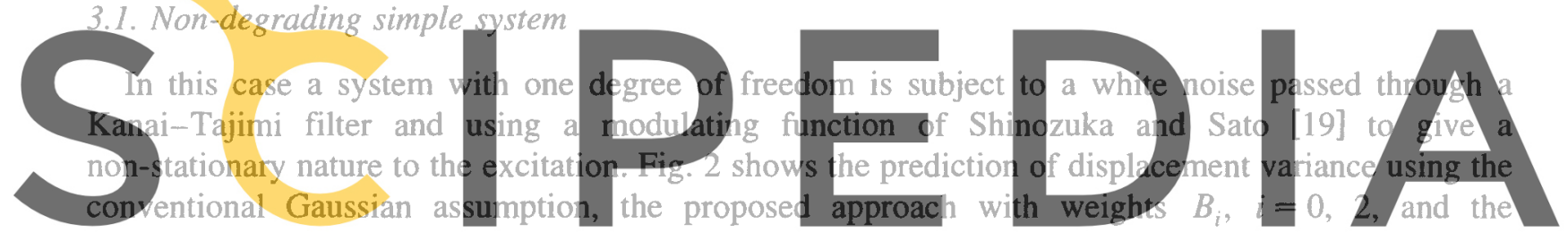

Register for free at https//www.scipedia.com to download the version without the watermark

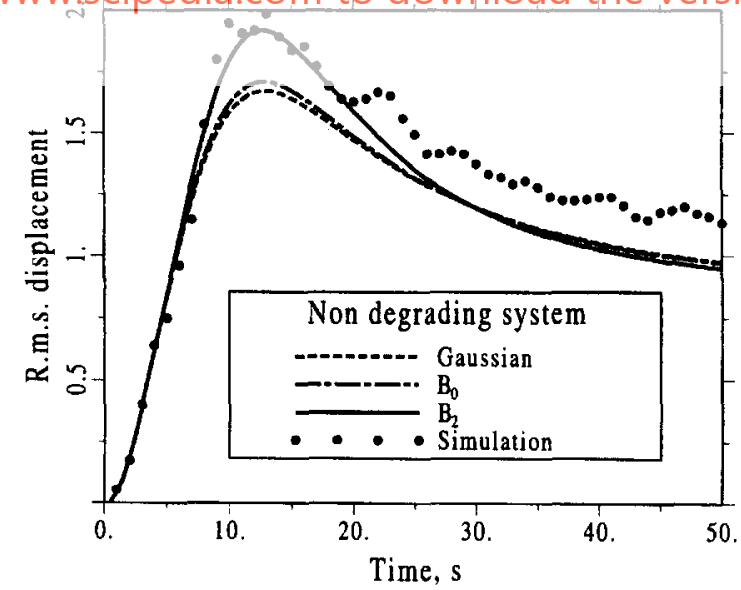

Fig. 2. Displacement response of a single degree of freedom non-degrading system using Gaussian and mixed distributions with $B_{0}$ and $B_{2}$. 


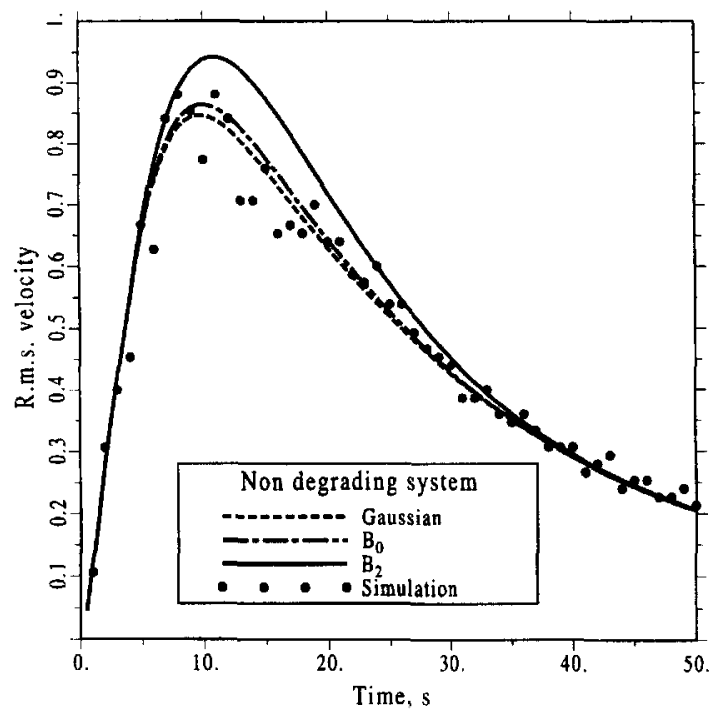

Fig. 3. Velocity response of a single degree of freedom non-degrading system using Gaussian and mixed distributions with $B_{0}$ and $B_{2}$.

simulation results. While the use of $B_{0}$ gives a slightly better solution than the Gaussian approach as expected, it is observed that a significant improvement is obtained with the application of the proposed method using the variance-based function $B_{2}$ in the zone of higher r.m.s. displacements, and similar results as those obrained with the Gaussian assumption in the final instants of this very long excitation. Since one of the main uses of the method of stochastic linearization is maximum responses from the knowledge of their variances, it is evident that the obtained with the present inethod has a relevant practical importance. With respect to the results of the estimated r.m.s. of the give accurate results in the whole time range, except in the zone of maximum values where they are Register for under- and heverestimated. by the conventignal and proposed approaches, respectively The ThW error of the proposed method in that region of the response is in this case about $6 \%$ on the sare side.

3.2. Two story shear building

The excitation in this case is also a filtered and modulated white noise. Fig. $4 a$ and $b$ show the results of the r.m.s. of the relative displacement of the two floors using the $B_{2}$ method. (In the rest of the examples this will be the only expression of the weighting coefficient that will be used unless otherwise stated.) In spite of the scatter of the simulation points, which is due to the fact that they were obtained in [8] with 50 samples only, the excellent improvement obtained with respect to the Gaussian method is again conspicuous. On the other hand, Fig. 5 shows the values of the function $B$ reached in the two stories. The maximum weights of the Dirac pulses are approximately $15 \%$ and $20 \%$, which are roughly similar to the value of the error of the Gaussian method.

\subsection{Degrading single degree of freedom systems}

Three cases of degradation were considered, by assigning a non-zero value to one of the three parameters in each case. Figs. 6 and 7 show that the results corresponding to the cases of stiffness 

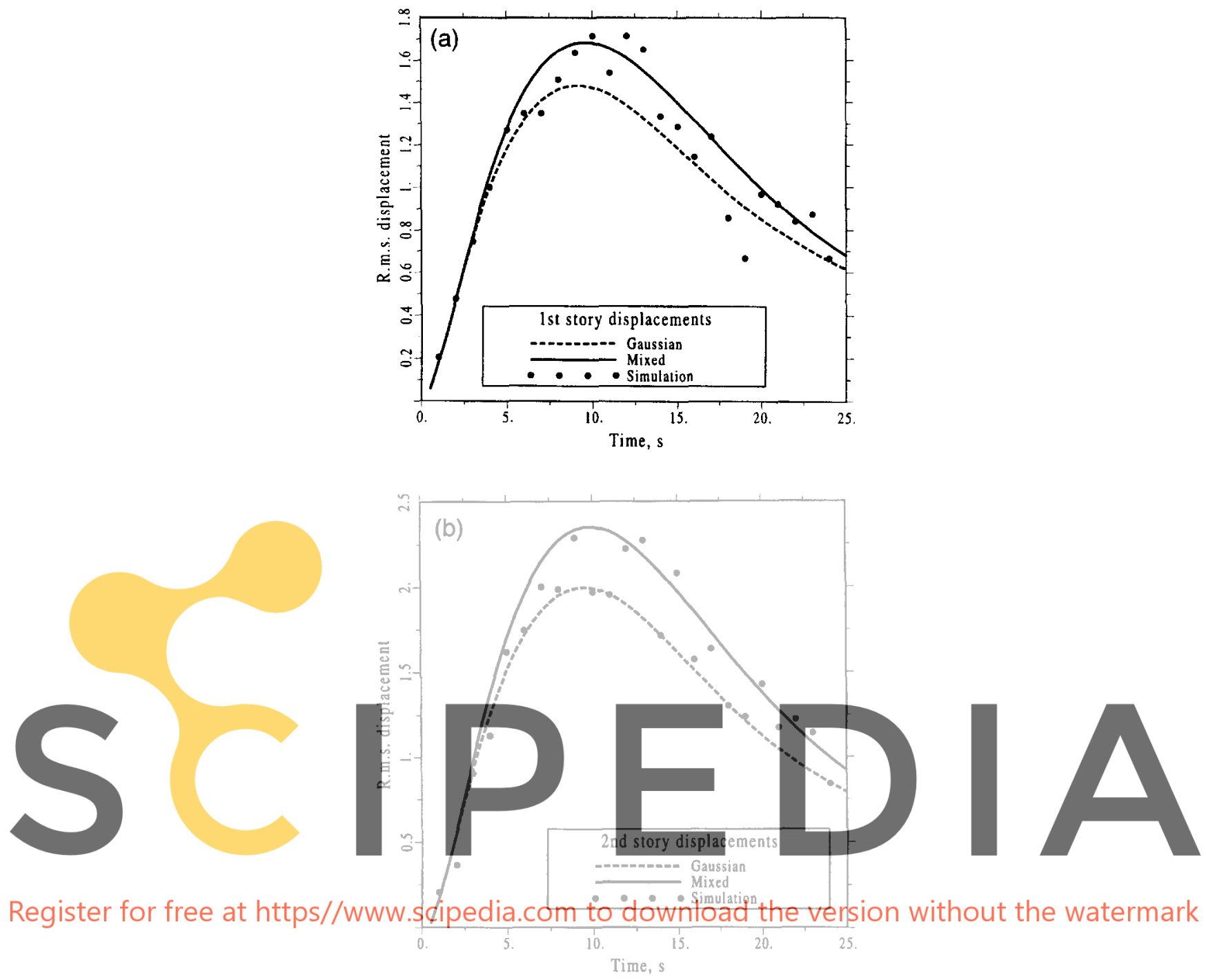

Fig. 4. Displacement response of the (a) first story and (b) second story of a shear building calculated with conventional (Gaussian) and proposed methods.

degradation with $\delta_{\eta}=0.2$ and both stiffness and strength deterioration with $\delta_{A}=0.05$. In both cases the agreement with the simulation results is again excellent in the zone of maximum responses, which are underestimated by the conventional approach.

The third case corresponds to a value of $\delta_{\nu}=0.2$. As Fig. 8 shows, a very large strength deterioration takes place in this case, as compared with that calculated in the previous example corresponding to $\delta_{A}=0.05$. In this case it is then expected that the displacement statistics obtained with the Gaussian hypothesis exhibit the poorest agreement with the experimental ones, due to the fact that more probability mass of the restoring force is allocated in the regions above and below the moving strength bounds as degradation increases. In fact, this is the example in [8] in which the 


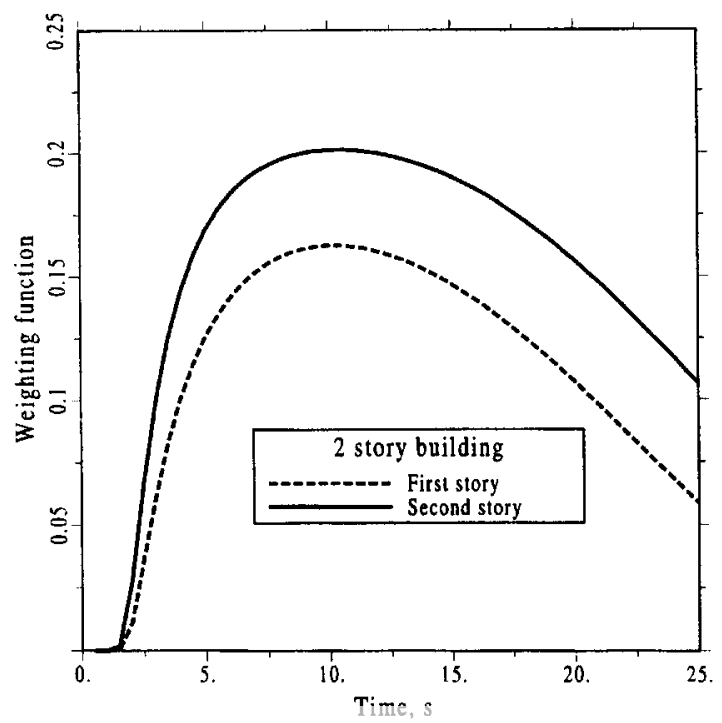

Fig. 5. Calculated values of the weighting function in the analysis of a two-story shear building.

greatest difference can be seen between the simulation and the results of the conventional method. In Fig. 9 it can be observed that the improvement with the proposed method is not as good as it was in the previous examples. In cases like this a stronger criterion for the correcting function $B$ appears to be necessary. Since one of the consequences of strength degradation is to produce a probability
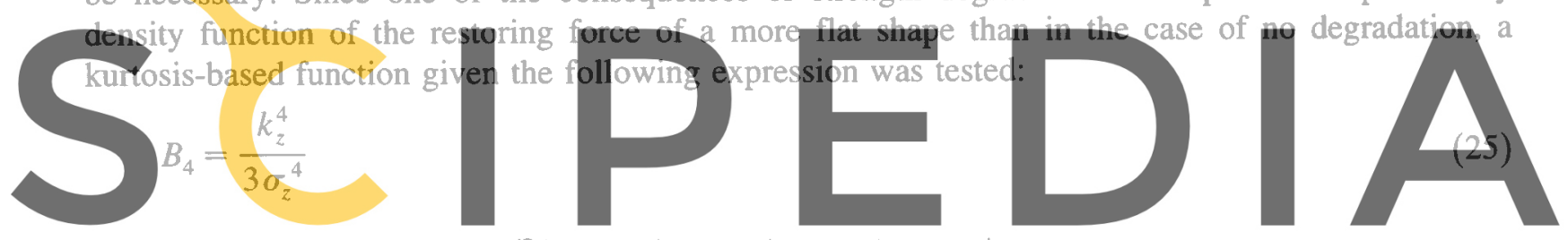

Register for free at https//www.scipedia.com to download the version without the watermark

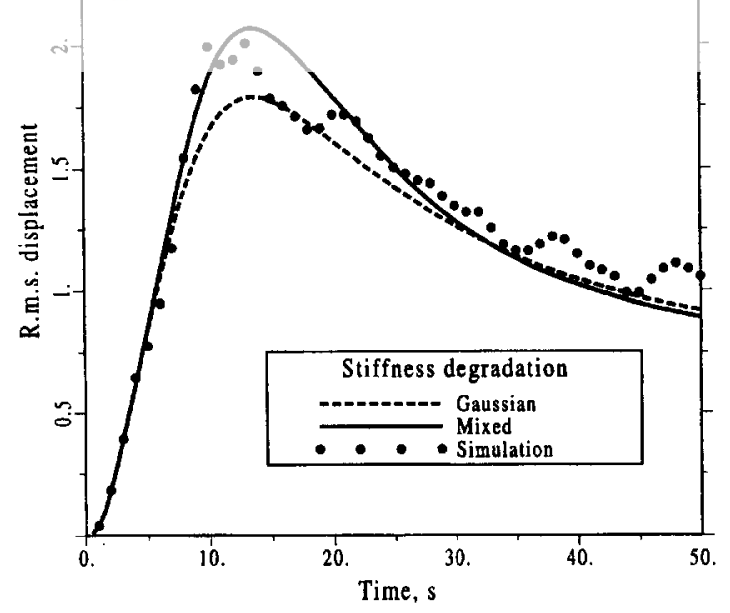

Fig. 6. Displacement response of a single degree of freedom system with stiffness degradation $\left(\delta_{\eta}=0.2\right)$. 


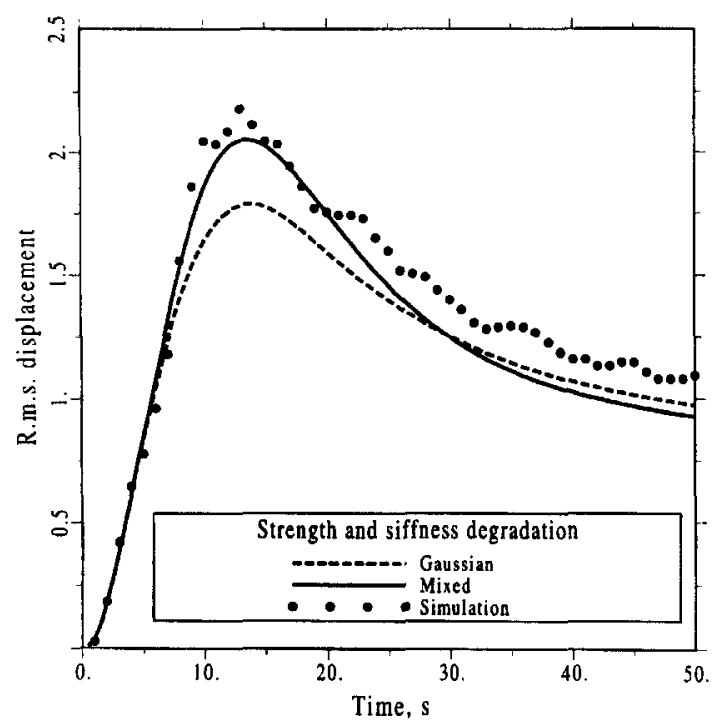

Fig. 7. Displacement response of a single degree of freedom system with strength and stiffness degradation $\left(\delta_{A}=0.05\right)$.

where

$$
k_{z}^{4}=\int_{z_{u}}^{\infty} z^{4} \varphi_{1}(z) \mathrm{d} z
$$

so that

$$
B_{4}=\frac{2}{3 \sqrt{\pi}} \Gamma^{c}\left(2.5, \frac{z_{u}^{2}}{2 \sigma_{z}^{2}}\right)
$$

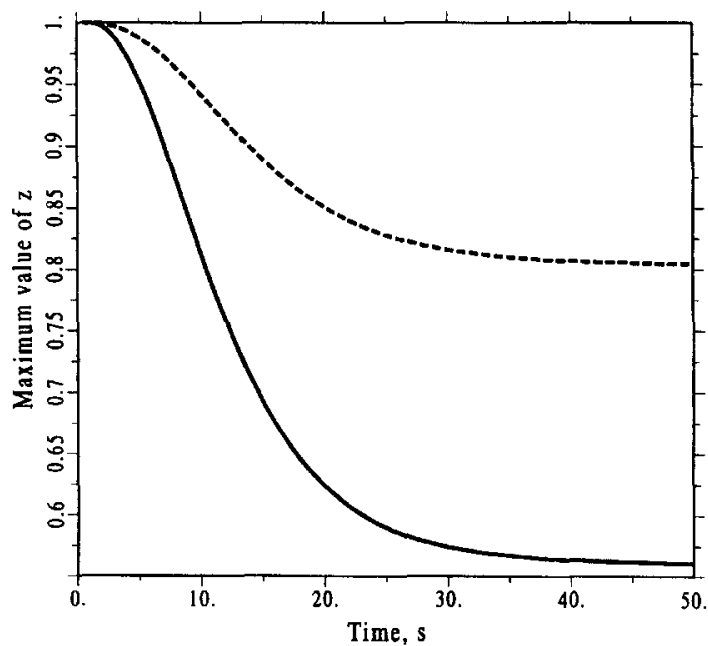

Fig. 8. Strength degradation reached by systems with $\delta_{A}=0.05$ (dashed line) and $\delta_{\nu}=0.20$ (solid line). 


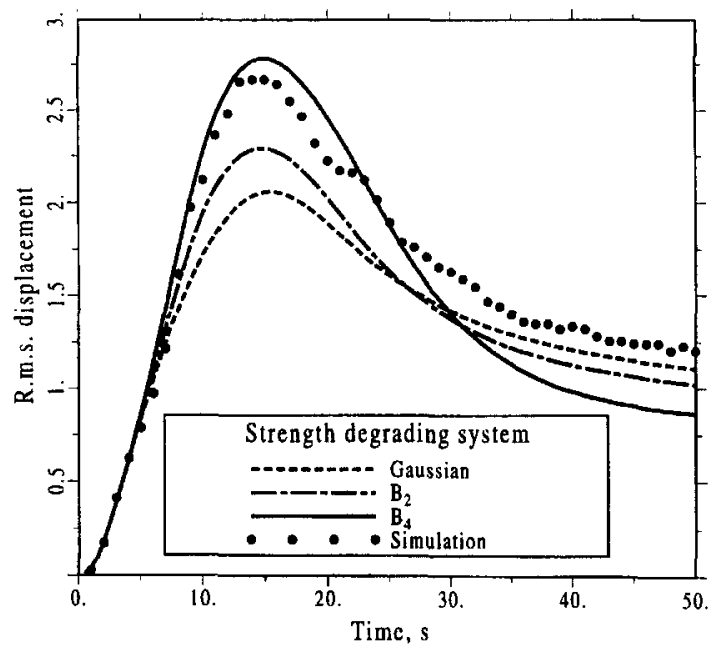

Fig. 9. Displacement response of a single degree of freedom system with strength degradation $\left(\delta_{\nu}=0.20\right)$ using variance $\left(B_{2}\right)$ and kurtosis $\left(B_{4}\right)$ criteria.

It is observed in Fig. 9 that the accuracy of the predicted maximum r.m.s. displacement using this stronger criterion is excellent, but that the estimation is affected in the last instants of the response.

From these numerical studies it is concluded that the variance-based weighting function can be used as the standard technique in the non-degrading case and in those degrading cases when there is not a large strength reduction, for which the kurtosis-based function gives better predictions of the maximum second moment displacement response.

\section{Conclusions and future research}

A method has been proposed to improve the equivalent linearization technique for random vibration analysis of hysteretic systems, by assuming that the non-Gaussianity of the restoring force can be expressed as a linear combination of Gaussian and Dirac densities. The combination is performed by means of weighting functions that depend on the degree of non-linearity reached by each non-linear degree of freedom of the structure at each time step. The main advantage of the proposed algorithm is that it preserves the computational simplicity of the Gaussian, conventional method without using complicated alternatives, because it maintains the use of the special properties of the Gaussian function. This allows the derivation of closed form expressions of the linearization coefficients in certain cases. In the present paper these expressions have been provided for the case of the Bouc-Wen-Baber hysteretic model.

Three possibilities for the calculation of the weighting functions have been tested. They are based respectively on the probability, variance and kurtosis contribution of the regions of the Gaussian density of the restoring force $z$ above the strength of the system. The numerical studies performed with both degrading and non-degrading structures show that the use of the second criterion overestimates somewhat the maximum standard deviation of the velocity, but it predicts them accurately in the ascending and descending branches of the non-stationary response. With respect to 
the displacement response, an excellent agreement with the simulation results was observed in the zone of maximum responses, and a prediction similar to that obtained by the conventional approach in the last instants of the response. In a special case when a very large strength degradation takes place, the use of the kurtosis-based weighting function gives an excellent adjustment with the simulation up to the instant of time corresponding to the maximum r.m.s. response, but the prediction after that time is affected. Nevertheless, since the estimation of maximum responses is dominated by the maximum value of its standard deviation, the importance of obtaining this with better accuracy is self-evident. For all these reasons it can be concluded that the proposed method represents a significant improvement with respect to the conventional method based on the assumption of Gaussian response, requiring the same computational effort.

Additional analyses are being conducted by the authors to study the prediction of the random response of other non-linear systems and the effectivity of the proposed method for the estimation of failure probabilities, for which the conventional Gaussian method is known to give non-conservative estimations at high excitation levels.

\section{Acknowledgements}

Financial supports for the realization of the present research at the Technical University of Catalonia has been received by the first author from the Colombian Science Institute "Colciencias" and the National University of Colombia. The support is gratefully acknowledged.

\section{References}

[1] Caughey, T.K., Equivalent linearization techniques. Journal of the Acoustical Society of America, 1963, 35, $1706-1711$.

[2] Iwan, W.D., A generalization of the concept of equivalent linearization. International Journal of Non-linear Mechanics, 1973, 8, 279-287.

[3] Atalik, T.S. and Utku, S., Stochastic linearization of multi-degree of freedom non-linear systems. Earthquake Engineering and Structural Dynamics, 1976, 4, 411-420.

[4] Lin, Y.K., Kozin, F., Wen, Y.K., Casciatti, F., Schuëller, G.I., Der Kiureghian, A., Ditlevsen O. and Vanmarcke, E.H., Methods of stochastic structural dynamics. Structural Safety, 1986, 3, 167-194.

[5] Roberts, J.B. and Spanos, P.D., Random Vibration and Statistical Linearization. John Wiley, Chichester, UK, 1990.

[6] Bouc, R., Forced vibration of mechanical systems with hysteresis (Abstract). In: Proc. Fourth Conf. on Non-linear Oscillation, Prague, Czechoslovakia, 1967.

[7] Wen, Y.K., Equivalent linearization for hysteretic systems under random excitation. Journal of Applied Mechanics, $1980,47,150-154$.

[8] Baber, T.T. and Wen, Y.K., Stochastic equivalent linearization for hysteretic, degrading, multistorey structures. Report UILU-ENG-80-2001, University of Illinois at Urbana-Champaign, 1980.

[9] Wen, Y.K. and Eliopoulos, D., Method for non-stationary random vibration of inelastic structures. Probabilistic Engineering Mechanics, 1994, 9, 115-123.

[10] Pires, J.E.A., Wen Y.K. and Ang, A.H.S., Stochastic analysis of liquefaction under earthquake loading. Civil Engineering Studies, SRS No. 504, University of Illinois at Urbana-Chąmpaign, 1983.

[11] Constantinou, M.C. and Tadjbakhsh, I.G., Hysteretic dampers in base isolation: random approach. Journal of Structural Engineering, 1985, 111, 705-721.

[12] Yang, J.N., Li, Z. and Vongchavalitkul, S., Stochastic hybrid control of hysteretic structures. Probabilistic Engineering Mechanics, 1994, 9, 125-133. 
[13] Thyagarajan, R.S. and Iwan, W.D., Performance characteristics of a widely used hysteretic model in structural dynamics. In: Proc. Fourth U.S. National Conf. on Earthquake Engineering, Palm Springs, 1990. EERI, Oakland, 1990, pp. 177-186.

[14] Casciatti, F. and Faravelli, L., Fragility Analysis of Complex Structural Systems. Research Studies Press, Taunton, UK, 1990.

[15] Pradlwarter, H.J. and Schuëller, G.I., The method of statistical equivalent linearization. In: Structural Dynamics Recent Advances, Springer-Verlag, Berlin, 1991.

[16] Schuëller, G.I., Pandey, M.D. and Pradlwarter, H.J., Equivalent linearization (EQL) in engineering practice for aseismic design. Probabilistic Engineering Mechanics, 1994, 9, 95-102.

[17] Kimura, K., Yasumuro, H. and Sakata, M., Non-Gaussian equivalent linearization for non-stationary random vibration of hysteretic system. Probabilistic Engineering Mechanics, 1994, 9, 15-22.

[18] Wen, Y.K. and Yeh, C.H., Biaxial and torsional response of inelastic structures under random excitation. Structural Safety, 1989, 6, 137-152.

[19] Shinozuka, M. and Sato, Y., Simulation of non-stationary random processes. Journal of the Engineering Mechanics Division, 1967, 93, 11-40. 